preprint

\title{
The Fermion Chern-Simons Gauge Theory of Fractional Quantum Hall Effect for Electromagnetic Polarization Tensor
}

\author{
Tae-Hyoung Gimm and Sung-Ho Suck Salk \\ Department of Physics, Pohang University of Science and Technology, Pohang 790-784, Korea
}

(October 23, 2018)

\begin{abstract}
Unlike an earlier theory, by avoiding both the electromagnetic gauge field shift and the assumption of the zero average of electromagnetic field fluctuation the fermion Chern-Simons gauge theory is reformulated to obtain mean field solutions and a self-consistent expression of the electromagnetic polarization tensor in terms of the composite fermion picture for the systems of fractional quantum Hall effect. Thus the newly derived electromagnetic polarization tensor is shown to depend on the residual (effective) magnetic field 'seen' by composite fermions rather than the statistical field, which differs from the earlier theory. The present theory reproduces the Hall conductance of fractional quantum Hall effect. The self-consistent picture of the composite fermion is maintained in all of our derivations: both the mean field solutions and the electromagnetic polarization tensor are described by the residual magnetic field seen by the composite fermions.

PACS numbers: 73.40.Hm, 71.27.+a, 11.15.-q
\end{abstract}

Typeset using REVTEX 


\section{INTRODUCTION}

Recently the fermion Chern-Simons theory has been of great interest to study the systems of fractional quantum Hall effect (FQHE). Based on the composite fermion theory initiated by Jain, the fermion Chern-Simons theory of the FQHE system is advanced by Lopez and Fradkin 2 a 3 and Halperin and coworkers. ${ }^{4}$ Jain 11 proposed the transformation of interacting 2-D electrons under an external magnetic field to composite fermions. To be more specific, he interpreted the Laughlin ground state 5 as a state in which the interacting electrons bind flux quanta to screen out part of the external magnetic field by fastening each electron with an even number of flux quanta and these composite fermions exactly fill an integer number of Landau levels.

Lopez and Fradkin 2 B proposed the fermion Chern-Simons theory of the FQHE by allowing fermions coupled to the statistical gauge field to form the composite fermions; the partition function is given by

$$
\mathcal{Z}(A)=\int \mathcal{D} \psi^{*} \mathcal{D} \psi \mathcal{D} a_{\mu} \exp (i \mathcal{S})
$$

where the action $S$ is explicitly,

$$
\begin{aligned}
\mathcal{S}= & \int d^{3} z\left\{\psi^{*}\left[i \frac{\partial}{\partial t}-e\left(A_{0}-a_{0}\right)+\mu\right] \psi(z)-\frac{1}{2 m}|[\nabla+i e(\mathbf{A}-\mathbf{a})] \psi(z)|^{2}+\right. \\
& \left.+\frac{\alpha}{2} \varepsilon^{\mu \nu \rho} a_{\mu} \partial_{\nu} a_{\rho}\right\}-\frac{1}{2} \int d^{3} z \int d^{3} z^{\prime}\left(|\psi(z)|^{2}-\bar{\rho}\right) V\left(z-z^{\prime}\right)\left(\left|\psi\left(z^{\prime}\right)\right|^{2}-\bar{\rho}\right),
\end{aligned}
$$

Here $\psi(z)$ is a second quantized Fermi field and $\mu$, the chemical potential. $A$ and $a$ are the electromagnetic vector potential and the statistical gauge potential respectively. $V$ is the pair interaction and $\bar{\rho}$, the average electron density. The resulting Chern-Simons 'Maxwell' equation of the $\mu=0$ component is

$$
\varepsilon^{i j} \partial_{i} a_{j}(x)=\frac{e}{\alpha} \rho(x)=b
$$

Here $b$ is the statistical 'magnetic' field and $\rho(x)$, the electron density. $\alpha$ is the Chern-Simons

(statistical) coupling constant given by $\alpha=\frac{e}{n \phi_{0}}$ or $\alpha=\frac{1}{2 \pi n}$ for $\hbar=e=c=1$ with the 
choice of an even number $n$ of the statistical flux quanta $\phi_{0}$. The action above involves the dynamics of a system of spinless interacting fermions coupled to both the electromagnetic and statistical (Chern-Simons) gauge fields.

In the present study we evaluate the effective action and present a generalized theory of electromagnetic response functions by avoiding the gauge shifts and the assumption of the zero average of external electromagnetic fluctuation, unlike the original theory of Lopez and Fradkin. 23 Both the mean field solutions and the electromagnetic polarization function are described by the residual (effective) magnetic field 'felt' by the composite fermions.

\section{MEAN FIELD SOLUTIONS AND ELECTROMAGNETIC RESPONSE FUNCTIONS}

In the fermion Chern-Simons theory, the statistical gauge field does not completely screen out the electromagnetic gauge field. Thus there exists an residual (effective) gauge field, that is, the unscreened portion of the electromagnetic gauge field, $A_{\mu}^{\mathrm{eff}}$,

$$
A_{\mu}^{\mathrm{eff}} \equiv A_{\mu}-a_{\mu}
$$

The effective (residual) magnetic field is then

$$
\vec{B}^{\mathrm{eff}}=\vec{\nabla} \times \vec{A}^{\mathrm{eff}}
$$

In the approach of Lopez and Fradkin, 2, 3 a small fluctuating electromagnetic field is introduced into the Chern-Simons term by allowing gauge field shifts. They also assumed the vanishing average of the external electromagnetic fluctuation for the evaluation of various

mean field solutions. In the following we avoid such gauge field shifts and the assumption of the vanishing average of the external electromagnetic fluctuation.

The Hubbard-Stratonovich transformation of the quartic fermion field term in (1.1) and the integration over the fermi fields yields the effective action, 目 3

$$
\mathcal{S}_{\text {eff }}=-i \operatorname{Tr} \ln \left(i D_{0}+\mu+\lambda-\frac{1}{2 m} \mathbf{D}^{2}\right)+\mathcal{S}_{\mathrm{CS}}\left[a_{\mu}\right]+\mathcal{S}[\lambda]
$$


where

$$
\mathcal{S}[\lambda]=-\int d^{3} z \lambda(z) \bar{\rho}+\frac{1}{2} \int d^{3} z \int d^{3} z^{\prime} \lambda(z) V^{-1}\left(z-z^{\prime}\right) \lambda\left(z^{\prime}\right)
$$

In order to obtain the classical configurations $\bar{a}_{\mu}(z) \equiv\left\langle a_{\mu}(z)\right\rangle$ and $\bar{\lambda}(z) \equiv\langle\lambda(z)\rangle$, we introduce the semiclassical (saddle point) approximation by requiring that $S_{\text {eff }}$ be stationary under small fluctuations;

$$
\left.\frac{\delta \mathcal{S}_{\text {eff }}}{\delta a_{\mu}(z)}\right|_{\bar{a}, \bar{\lambda}}=0 \quad \text { and }\left.\quad \frac{\delta \mathcal{S}_{\text {eff }}}{\delta \lambda(z)}\right|_{\bar{a}, \bar{\lambda}}=0
$$

By introducing $a_{\mu}=A_{\mu}-A_{\mu}^{\mathrm{eff}}$ into the Chern-Simons term of (2.3), the equations of motion from the variation of $\mathcal{S}_{\text {eff }}$ with respect to $a_{\mu}(z)$ and $\lambda(z)$ in (2.5) are obtained as

$$
\left\langle j_{\mu}(z)\right\rangle-\frac{1}{n \phi_{0}} \varepsilon^{\mu \nu \rho}\left\{\left\langle\partial_{\nu} A_{\rho}(z)\right\rangle-\left\langle\partial_{\nu} A_{\rho}^{\mathrm{eff}}(z)\right\rangle\right\}=0
$$

and

$$
\left\langle j_{0}\right\rangle-\bar{\rho}+\int d^{3} z^{\prime} V^{-1}\left(z-z^{\prime}\right)\left\langle\lambda\left(z^{\prime}\right)\right\rangle=0 .
$$

Here it is of note that the above equation of motion (2.6) differs from that of Lopez and Fradkin; : the first term in their equation (3.10) in Ref. 2 is given by the difference between the statistical field strength tensor $\mathcal{F}^{\mu \nu}$ and the electromagnetic field strength tensor $F^{\mu \nu}$.

The condition for the uniform liquid state is obtained from (2.7),

$$
\left\langle j_{0}\right\rangle=\bar{\rho},
$$

for $\langle\lambda(z)\rangle=0$. From (2.1) and (2.2), the Chern-Simons (statistical) magnetic field is given by

$$
\vec{b}=\vec{\nabla} \times \vec{a}=\vec{B}-\vec{B}^{\text {eff }}
$$

Using (2.8) and (2.9) for (2.6) and setting $\mu=0$, we find the uniform average statistical magnetic field,

$$
\langle b\rangle=n \phi_{0} \bar{\rho}
$$


From (2.9) and (2.10) the effective (remaining) external magnetic field is then

$$
B^{\mathrm{eff}}=B-\langle b\rangle=B-n \phi_{0} \bar{\rho} .
$$

In coincidence with the Jain's composite fermion picture, each electron is now assumed to be dressed by an even number of statistical (Chern-Simons) flux quanta, $n=2 m$ with $m$, an integer. The number of effective (residual) flux quanta, $N_{\phi}^{\mathrm{eff}}$ is then from (2.11) above,

$$
N_{\phi}^{\mathrm{eff}}=N_{\phi}-2 m N_{e}
$$

Here $N_{\phi}$ is the total number of applied (external) flux quanta and $N_{e}$, the total number of electrons. From the filling fraction defined by $\nu=N_{e} / N_{\phi}$ and the relation (2.12), we readily obtain the fractional filling factor,

$$
\nu=\frac{p}{2 m p+1}
$$

with $p=\frac{N_{e}}{N_{\phi}^{\text {ett }}}$, and the effective Landau gap,

$$
\hbar \omega_{c}^{\mathrm{eff}}=\frac{\hbar \omega_{c}}{2 m p+1} .
$$

As shown above, we were able to avoid the gauge field shifts and the assumption of the vanishing average of fluctuating electromagnetic field in order to reproduce various mean field solutions: the uniform liquid state, the fractional filling fraction, and the effective Landau gap.

In order to obtain the linear response functions, we now consider the Gaussian fluctuations around the uniform classical values; $\delta A_{\mu}^{\mathrm{eff}}(z)$ and $\delta \lambda(x)$ are taken to be small fluctuations around the mean (classical) values of the effective (residual) field $A^{\text {eff }}$ and the collective mode $\lambda$, that is, $A_{\mu}^{\mathrm{eff}} \rightarrow\left\langle A_{\mu}^{\mathrm{eff}}\right\rangle+\delta A_{\mu}^{\mathrm{eff}}$ and $\lambda \rightarrow\langle\lambda\rangle+\delta \lambda$. In this spirit of the linear response theory the effective action to quadratic order is written,

$$
\begin{aligned}
\mathcal{S}^{(2)}= & \frac{1}{2} \int d^{3} x d^{3} y \delta A_{\mu}^{\mathrm{eff}}(x) \Pi_{\mu \nu}(x, y) \delta A_{\nu}^{\mathrm{eff}}(y)+ \\
& +\mathcal{S}_{\mathrm{CS}}\left[A_{\mu}-A_{\mu}^{\mathrm{eff}}-\delta_{\mu 0} \delta \lambda\right]+\frac{1}{2} \int d^{3} z d^{3} z^{\prime} \delta \lambda(z) V^{-1}\left(z-z^{\prime}\right) \delta \lambda\left(z^{\prime}\right),
\end{aligned}
$$


after the shift of the scalar bose field is made. The above expression is different from that of Lopez and Fradkin 23 in that $\Pi_{\mu \nu}$ is the polarization tensor associated with the residual gauge field, but not with the statistical gauge field. The effective action involving the quadratic term above is then equivalent to the one-loop bubble contributions associated with the effective field $A_{\mu}^{\mathrm{eff}}$.

The expansion of the Chern-Simons term around $\langle\lambda\rangle=0$ yields

$$
\begin{aligned}
\mathcal{S}_{\mathrm{CS}}\left[A_{\mu}-A_{\mu}^{\mathrm{eff}}-\delta_{\mu 0} \delta \lambda\right]= & \mathcal{S}_{\mathrm{CS}}\left[A_{\mu}-A_{\mu}^{\mathrm{eff}}\right]+ \\
& -\frac{\alpha}{2} \int d^{3} z \delta \lambda(z)\left(B(z)-B^{\mathrm{eff}}(z)\right)+(\text { h.o. }) \\
= & \mathcal{S}_{c s}\left[A_{\mu}-A_{\mu}^{\mathrm{eff}}\right]-\frac{\alpha}{2} \int d^{3} z \delta \lambda(z) b(z)+(\text { h.o. }) .
\end{aligned}
$$

A scalar (collective) bose field term is, from (2.15) and (2.16),

$$
S^{(2)}[\delta \lambda]=\frac{\alpha}{2} \int d^{3} z \delta \lambda(z) b(z)+\frac{1}{2} \int d^{3} z d^{3} z^{\prime} \delta \lambda(z) V^{-1}\left(z-z^{\prime}\right) \delta \lambda\left(z^{\prime}\right) .
$$

After the Gaussian integration over the collective bose field $\lambda$, the effective action (2.15) becomes

$$
\begin{aligned}
\mathcal{S}_{\text {eff }}^{(2)}= & \frac{1}{2} \int d^{3} x d^{3} y \delta A_{\mu}^{\mathrm{eff}}(x) \Pi_{\mu \nu}(x, y) \delta A_{\nu}^{\mathrm{eff}}(y)+ \\
& +\mathcal{S}_{\mathrm{SC}}\left[A_{\mu}-A_{\mu}^{\mathrm{eff}}\right]+ \\
& -\frac{\alpha^{2}}{2} \int d^{3} z \int d^{3} z^{\prime} b(z) V\left(z-z^{\prime}\right) b\left(z^{\prime}\right) .
\end{aligned}
$$

The polarization tensor in (2.18) can be expressed in the low energy limit in terms of three gauge invariant tensors $E_{\text {eff }}^{2}, B_{\text {eff }}^{2}$ and Chern-Simons terms which are associated with the effective gauge field $A^{\text {eff }}$, not with the statistical gauge field. Thus the coefficients of these tensors are namely the dielectric constant $\epsilon^{0}$, diamagnetic susceptibility $\chi^{0}$, and Hall conductance $\sigma_{x y}^{0}$ in association with the response of the system of the composite fermions to the weak perturbation of the residual field, $\delta A_{\mu}^{\mathrm{eff}}$.

In order to integrate out the statistical gauge field, we rewrite (2.18),

$$
\mathcal{S}_{\text {eff }}^{(2)}=\frac{1}{2} \int d^{3} x d^{3} y \delta\left(A_{\mu}(x)-a_{\mu}(x)\right) \Pi_{\mu \nu}(x, y) \delta\left(A_{\nu}(y)-a_{\nu}(y)\right)+
$$




$$
\begin{aligned}
& +\mathcal{S}_{\mathrm{CS}}\left[a_{\mu}\right]+\frac{1}{2 \beta}\left(\partial_{\mu} a^{\mu}\right)^{2}+ \\
& -\frac{\alpha^{2}}{2} \int d^{3} z \int d^{3} z^{\prime} b(z) V\left(z-z^{\prime}\right) b\left(z^{\prime}\right),
\end{aligned}
$$

where we introduced a gauge fixing term, $\frac{1}{2 \beta}\left(\partial_{\mu} a^{\mu}\right)^{2}$ in order to avoid singularity in the inverse matrix of the quadratic term in $a_{\mu}$ in momentum space.

We obtain from (2.19) the following matrix for the quadratic term in $a_{\mu}$ in momentum space representation

$$
M=\left[\begin{array}{lll}
\mathbf{q}^{2} \Pi_{0}+\frac{\omega^{2}}{\beta} & \omega q_{1} \Pi_{0}+i q_{2}\left(\Pi_{1}+\alpha\right)-\frac{\omega q_{1}}{\beta} & \omega q_{2} \Pi_{0}-i q_{1}\left(\Pi_{1}+\alpha\right)-\frac{\omega q_{2}}{\beta} \\
\omega q_{1} \Pi_{0}-i q_{2}(\Pi+\alpha)-\frac{\omega q_{1}}{\beta} & \omega^{2} \Pi_{0}+q_{2}^{2}\left(\Pi_{2}-\alpha^{2} V\right)+\frac{q_{1}^{2}}{\beta} & -q_{1} q_{2}\left(\Pi_{2}-\alpha^{2} V\right)-i \omega\left(\Pi_{1}+\alpha\right)+\frac{q_{1} q_{2}}{\beta} \\
\omega q_{2} \Pi_{0}+i q_{1}\left(\Pi_{1}+\alpha\right)-\frac{\omega q_{2}}{\beta} & -q_{1} q_{2}\left(\Pi_{2}-\alpha^{2} V\right)+i \omega\left(\Pi_{1}+\alpha\right)+\frac{q_{1} q_{2}}{\beta} & \omega^{2} \Pi_{0}+q_{1}^{2}\left(\Pi_{2}-\alpha^{2} V\right)+\frac{q_{2}^{2}}{\beta}
\end{array}\right]
$$

The determinant of the matrix above is then

$$
\operatorname{det} M=\frac{D(\omega, \mathbf{q})}{\beta}\left(\omega^{2}+\mathbf{q}^{2}\right)^{2}
$$

where

$$
D(\omega, \mathbf{q}) \equiv \Pi_{0}^{2} \omega^{2}-\left(\Pi_{1}+\alpha\right)^{2}+\Pi_{0}\left(\Pi_{2}-\alpha^{2} V\left(\mathbf{q}^{2}\right)\right) \mathbf{q}^{2}
$$

with $\Pi_{l}=\Pi_{l}(\omega, \mathbf{q})$ with $l=0,1,2$.

The Gaussian integral can now be performed by taking the inverse of the above $3 \times 3$ matrix $M$. After the Gaussian integration over the statistical gauge field, the effective action for the electromagnetic fluctuation is

$$
\mathcal{S}_{\text {eff }}^{\mathrm{EM}}=\frac{1}{2} \int d^{3} x \int d^{3} y \delta A_{\mu}(x) K^{\mu \nu}(x, y) \delta A_{\nu}(y) .
$$

Here the components of the above electromagnetic polarization tensor $K^{\mu \nu}$ in momentum space are obtained as

$$
\begin{aligned}
& K_{00}=\mathbf{q}^{2} K_{0}(\omega, \mathbf{q}), \\
& K_{0 j}=\omega q_{j} K_{0}(\omega, \mathbf{q})+i \epsilon_{j k} q_{k} K_{1}(\omega, \mathbf{q}), \\
& K_{j 0}=\omega q_{j} K_{0}(\omega, \mathbf{q})-i \epsilon_{j k} q_{k} K_{1}(\omega, \mathbf{q}), \\
& K_{i j}=\omega^{2} \delta_{i j} K_{0}(\omega, \mathbf{q})-i \epsilon_{i j} \omega K_{1}(\omega, \mathbf{q})+\left(\mathbf{q}^{2} \delta_{i j}-q_{i} q_{j}\right) K_{2}(\omega, \mathbf{q}),
\end{aligned}
$$


where

$$
\begin{aligned}
& K_{0}(\omega, \mathbf{q})=-\frac{\alpha^{2} \Pi_{0}}{D(\omega, \mathbf{q})} \\
& K_{1}(\omega, \mathbf{q})=\alpha+\alpha^{2} \frac{\left(\alpha+\Pi_{1}\right)}{D(\omega, \mathbf{q})}+\alpha^{3} V(q) \mathbf{q}^{2} \frac{\Pi_{0}}{D(\omega, \mathbf{q})} \\
& K_{2}(\omega, \mathbf{q})=-\alpha^{2} \frac{\Pi_{2}+V(\mathbf{q})\left(\omega^{2} \Pi_{0}^{2}-\Pi_{1}^{2}+\mathbf{q}^{2} \Pi_{0} \Pi_{2}\right)}{D(\omega, \mathbf{q})} .
\end{aligned}
$$

In the limit of small $\omega$ and $\mathbf{q}, \Pi_{l}(\omega, \mathbf{q})$ 's with $l=0,1$, and 2 are obtained as

$$
\begin{aligned}
& \Pi_{0}(0,0)=\frac{p M}{2 \pi B^{\mathrm{eff}}}=\frac{p M}{2 \pi(B-b)} \\
& \Pi_{1}(0,0)=\frac{p}{2 \pi} \\
& \Pi_{2}(0,0)=-\frac{p}{2 \pi M}
\end{aligned}
$$

with $M$ is the electron mass. $K_{1}$ in 2.25$)$ leads to

$$
\lim _{\mathbf{q} \rightarrow 0} \lim _{\omega \rightarrow 0} K_{1}(\omega, \mathbf{q})=\frac{\alpha \Pi_{1}(0,0)}{\alpha+\Pi_{1}(0,0)} .
$$

Using (2.26) and $\alpha=\frac{1}{2 \pi n}$ with $n=2 m$ in (2.27) above, we reproduce the Hall conductivity

$$
K_{1}(0,0)=\sigma_{x y}=\nu
$$

which is a fractional multiple of $\frac{e^{2}}{\hbar}$.

As shown in (2.26) above $\Pi_{0}(0,0)$ is seen to be different from the result of Lopez and

Fradkin, that is, $\Pi_{0}(0,0)=\frac{p M}{2 \pi b_{\text {eff }}}$ with $b_{\text {eff }}$ defined as $a_{1}(x)=-b_{\text {eff }} x_{2}$ and $a_{2}=0$ in the Landau gauge (or $\mathcal{A}_{1}(x)=-\mathcal{B}_{\text {eff }} x_{2}$ and $\mathcal{A}_{2}(x)=0$ using their notation for the statistical gauge potential $\mathcal{A}_{\mu}\left(=a_{\mu}\right)$ as shown in eq. (B3) in Ref. 2). Thus unlike the theory of Lopez and Fradkin, 3 the electromagnetic polarization tensor $K^{\mu \nu}$ is described by the effective magnetic field $B^{\text {eff }}$ seen by the composite fermions but not by the statistical magnetic field $b$.

\section{CONCLUSION}

In the present study we treated the fermion Chern-Simons theory of FQHE in a straightforward manner, by avoiding the gauge field shifts. Further the assumption of the zero 
average of electromagnetic field fluctuations was not necessary to reproduce various mean field solutions: the uniform liquid state, the effective filling fraction, and the effective Landau gap. Unlike the theory of Lopez and Fradkin, 晛 the derived electromagnetic polarization tensor is described by the residual (effective) magnetic field rather than the statistical field, thus maintaining the self-consistent picture of the composite fermion. The present approach also reproduces the Hall conductance of the fractional quantum Hall effect. In all of our derivations the self-consistency of the composite fermion picture was maintained: both the mean field solutions and the electromagnetic polarization tensor were shown to depend on the residual magnetic field seen by the composite fermions.

\section{ACKNOWLEDGEMENT}

One of the authors (SHSS) acknowledges the generous supports of Korean Ministry of Education under the BSRI program and the Center for Molecular Sciences at Korea Advanced Institute of Science and Technology. He is also grateful to Pohang University of Science and Technology (Program P95002) for a partial financial support. 


\section{REFERENCES}

${ }^{1}$ J. K. Jain, Phys. Rev. Lett. 63, 199 (1989); Phys. Rev. B 40, 8079 (1989); Phys. Rev. B 41, 7653 (1990); Adv. Phys. 41, 105 (1992); Comments Cond. Matt. Phys. 16, 307 (1993).

${ }^{2}$ A. Lopez and E. Fradkin, Phys. Rev. B 44, 5246 (1991).

${ }^{3}$ A. Lopez and E. Fradkin, Phys. Rev. B 47, 7080 (1993).

${ }^{4}$ B. I. Halperin, P. A. Lee, and N. Read, Phys. Rev. B 47, 7312 (1993); S. H. Simon and B. I. Halperin, Phys. Rev. B 48, 7368 (1993); Phys. Rev. B 50, 1807 (1994); S. He, S. H. Simon, and B. I. Halperin, Phys. Rev. B 50, 1823 (1994).

${ }^{5}$ R. B. Laughlin, Phys. Rev. Lett. 50, 1395 (1983); The Quantum Hall Effect edited by R. Prange and S. Girvin (Springer-Verlag, New York 1989).

* e-mail: thgim@anyon.postech.ac.kr

$\dagger$ e-mail: salk@vision.postech.ac.kr 